\title{
Study of QCD phase using surface operators
}

\author{
Alexander Molochkov ${ }^{* \dagger}$ \\ School of Biomedicine, Far Eastern Federal University \\ E-mail: molochkov.alexanderegmail.com

\section{Valdimir Goy} \\ School of Biomedicine, Far Eastern Federal University \\ E-mail: vovagoy@gmail.com
}

\begin{abstract}
The most important probes for the phase states of a four-dimensional gauge field theory are the Wilson and t'Hooft line operatros that are defined on one-dimensional curves in the space-time. For example, these line-operators define order parameters for the confinement-deconfinement phase transition of the QCD vacuum. However, for more detail understanding of four-dimensional gauge field theory dynamics and vacuum topology we need additional probes expressed by operators defined on the subspaces with higher dimensions. Possible candidates are operators that are defined on the two-dimensional surface in the four-dimensional space-time. In the present work the surface operator in the lattice QCD is studied. The Witten parameter dependence on the surface area and volume studied in confinement and deconfinement phases.
\end{abstract}

XV International Conference on Hadron Spectroscopy-Hadron 2013

4-8 November 2013

Nara, Japan

* Speaker.

${ }^{\dagger}$ This project is supported by the Far Eastern Federal University. 


\section{Introduction}

Confinement in the QCD is the fundamental property of hadron matter that define spectra of hadrons. Quark confinement, in particular, is characterized by a linear potential interaction at large distances. However, to date there is no clear understanding how the color gauge interaction leads to this kind of quark potential. One of the promising explanations was obtained within the dual superconductor model. In the model the condensation of electric charges to the Cooper pairs is substituted by the chromomagnetic monopoles condesnation [四]. As the result, the chromoelectric field between color charges is compressed to flux tubes, what leads to the linear quark potential at the large distances. This explanation implies that the confinement-deconfinement phase transition is accompanied by vacuum phase transition associated with the condensation of chromomagnetic monopoles. Thus, one can assume existence of additional order parameters that are sensitive to the monopoles condensation. One of the candidates is the surface operator [B] proposed by Witten [四, []. It determined via divergence of the chromomagnetic field through closed surface. The parameter dependence on the surface area can reproduce the behavior of the Wilson loop. For example an infinite temporal surface can be considered as spatial Wilson loop averaged over the imaginary time component. It is expected, also, that the monople condensations affects the dependence on the volume covered by the surface. Thus, study of the surface operator volume dependence can clarify the role of the monopole condensation in the confinement-deconfinement phase transition.

In non-abelian pure gauge theories, expectation values of large surface operators is difficult to compute through direct Monte-Carlo simulation, because the signal-to-noise ratio is very rapidly

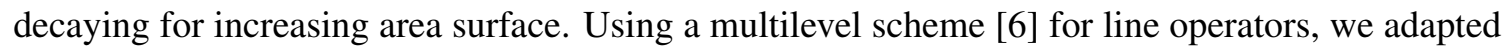
it to deal with surface operators when area exceeds $1 \mathrm{fm}^{2}$. The Witten parameter was numerically studied within the $S U$ (2) - gluodynamics using multilevel and multi-hit algorithms for the sake of statistical confidence in our previous papers [ [ $[$, [ ] It was shown that at the deconfinement phase the temporal surface operator exhibits nontrivial area dependence. In the confinement phase the operator is trivial with no area and volume dependence. It is shown also that the spatial surface operator exhibits the same phase behavior.

In the present paper we study volume dependence of the surface operator within direct MonteCarlo simulation.

\section{Surface operator on the lattice}

In general case the surface operator can be defined by the following expression:

$$
W=e^{i \kappa \int_{S} F_{i j} d \sigma^{i j}}
$$

where $F_{i k}$ - the gauge field tensor, $d \sigma_{i k}$ - surface element (here we do not distinguish between upper and lower indexes, because all calculations are performed in Euclidean space-time after Wick rotation), $i, k=1,2,3$ - indexes of the space-time directions. The field flow through a lattice plaquette can be related with the plaquette angle $\theta_{p}$ as follows:

$$
\kappa \int_{S} F_{i j} d \sigma^{i j}=\theta_{p}
$$


Thus, we define the surface operator on the lattice as follows:

$$
W_{p}(S)=R e \prod_{S} e^{i \theta_{p}}
$$

The phase of a wave function is changing by the plaquette angle value by moving along the contour of the plaquette. This phase related with chromomagnetic field flow through plaquette surface as follows:

$$
\kappa \int_{S} \mathbf{H} \cdot d \mathbf{S}=\kappa \oint \mathbf{A} \cdot d \mathbf{l}=\theta_{p}
$$

where integration over $d \mathbf{l}$ carried out on a path covering the area $S$. This equation provides a simple connection of the surface and line operators in the trivial vacuum.

In this work we consider pure gauge field theory with $S U(2)$ group symmetry broken up to $U(1)$. Thus the $\theta_{p}$ related with $F_{\mu \nu}$ as follows:

$$
F_{p}=\widehat{1} \cos \theta_{p}+\imath n_{i} \sigma_{i} \sin \theta_{p},
$$

where $n_{i}$ - vector on the unit sphere, $\sigma_{i}$ - Pauli matrices, $F_{p}$ is a value of the gauge field tensor $F_{\mu v}$ on the plaquette. Thus, for the $\theta_{p}$ we can write the following expression:

$$
\theta_{p}=\arccos \left(\frac{1}{2} \operatorname{Tr} F_{p}\right) .
$$

All phases are calculated on the surface of the three dimension cube in the space-time. The function $\arccos (x)$ is defined within the range $[0, \pi]$. In the gauge group $U(1)$ the range of variation of the angle is $[0,2 \pi]$. Thereby, on the one side of cube the phase is selected as $\arccos \left(\frac{1}{2} \operatorname{Tr} F_{p}\right)$, on the opposite side as $\arccos \left(\frac{1}{2} \operatorname{Tr} F_{p}\right)+\pi$.

In lattice calculations we use the link variable $U_{i j} \in S U(2)$, where $i, j$ is number of lattice sites, located at the ends of the link. Variable $U_{i j}$ related with $A_{\mu}$ as follows:

$$
U_{i j}=e^{l g_{0} A_{\mu} a}
$$

where $a$ is distance between sites and $A_{\mu}$ is taken at the middle of the link $i j$. According to Wilson [Q] action for pure gauge theory can be written as follows:

$$
\begin{aligned}
& S=\sum_{\square} S_{\square}, \\
& S_{\square}=\beta\left[1-\frac{1}{2} \operatorname{Re} \operatorname{Tr}\left(U_{i j} U_{j k} U_{k l} U_{l i}\right)\right],
\end{aligned}
$$

where $\beta=4 / g_{0}^{2}$ and $\square$ is a plaquette. The partition function is

$$
Z=\int(d U) e^{-S(U)}
$$

Any observed value of a physical quantity $A$ we can calculated using following expression:

$$
\langle A\rangle=Z^{-1} \int(d U) A(U) e^{-S(U)},
$$

where $A(U)$ is physical quantity calculated on the lattice configurations $U$ and the integration is over all configurations with weight equal $e^{-S(U)}$. 


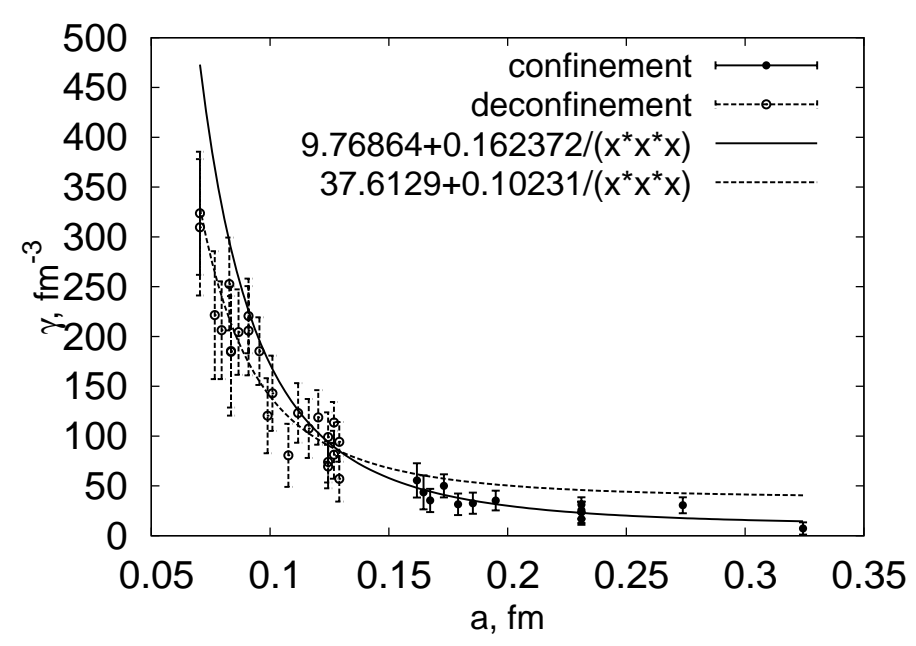

Figure 1: Dependence of the volume coefficient $\gamma$ on the lattice spacing. Solid line shows fit of the coefficient dependence in the confinement phase, dashed line is the fit in the deconfinement phase.

\section{Results}

To extract area and volume dependence of the surface operator we fit the obtained data by the following expression:

$$
W_{p}(S, V)=e^{-\sigma S-\gamma V},
$$

where $\sigma$ is area coefficient, $\gamma$ is volume coefficient, $S$ is the surface area, $V$ is volume covered by the surface. The parameter values are obtained with the help of minuit2 library from ROOT ${ }^{1}$ package. We parametrize the area and volume coefficients as follows:

$$
\begin{array}{r}
\sigma(a)=\sigma_{p h}+\sigma_{d i v} / a^{2} \\
\gamma(a)=\gamma_{p h}+\gamma_{d i v} / a^{3}
\end{array}
$$

where $\sigma_{p h}$ and $\gamma_{p h}$ are the physical coefficients and $\sigma_{d i v}$ and $\gamma_{d i v}$ are the coefficients of the divergent part of area and volume law.

Dependence of $\gamma$ on lattice spacing $a$ has different asymptotic at large $a$ in different phases (see figure 田. Numerical fit shows that $\gamma_{p h} \simeq 0$ in confinement phase and $\gamma_{p h} \neq 0$ in deconfinement case for spatial surface (see figure [). It allows to consider $\gamma_{p h}$ as an order parameter for the confinement-deconfinement phase transition, however large statistical errors do not allow to make definite conclusion.

In conclusion we can say following: 1) volume law of the spatial surface operator points on the possible phase transition associated with the chromomagnetic monopole condensation; 2) more definite conclusion about volume dependence of the spatial surface needs more precise numerical study.

\footnotetext{
${ }^{1}$ See http://root.cern.ch/drupal/
} 

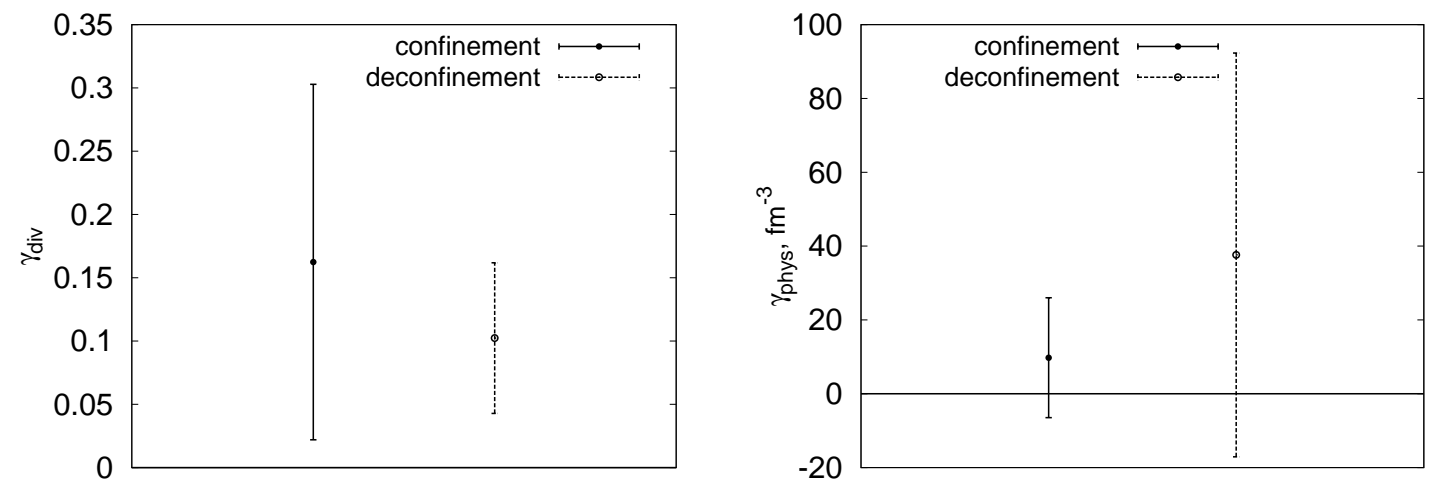

Figure 2: Values of the volume coefficients $\gamma_{d i v}$ and $\gamma_{p h}$ coefficients in the confinement and deconfinement phases for the spatial surface

\section{Acknowledgments}

We are thankful to Valetine Zakharov, Pavel Buividovich, Viktor Braguta, Maxim Chernodoub and Vitaly Bornyakov for interesting and useful discussions. We are very grateful to the late Mikhail Polikarpov for the inspiration and constant support of this work.

\section{References}

[1] M. N. Chernodub and M. I. Polikarpov, arXiv:hep-th/9710205 [hep-th] (1997).

[2] G. 't Hooft, Nuclear Physics B 190, 455 (1981).

[3] S. Gukov, "Phases of gauge theories and surface operators" the talk presented at the workshop "Prestrings", 11-15 August, 2008, Zürich, Switzerland.

[4] S. Gukov and E. Witten, arXiv:hep-th/0612073v2 [hep-th] (2007).

[5] S. Gukov and E. Witten, arXiv:0804.1561 [hep-th] (2008).

[6] M. Lüscher and P. Weisz, JHEP 0109, 010 (2001), arXiv:hep-lat/0108014 [hep-lat].

[7] A.V.Molochkov, V.A.Goy, PoS(LATTICE 2013) 359

[8] A.V.Molochkov, V.A.Goy, PoS(Confinement X) 313

[9] K. Wilson, Phys. Rev. D 10, 2445 (1974).

[10] M. Creutz, Quarks, gluons and lattice Cambridge University Press, Cambridge, 1983.

[11] G. Parisi, R. Petronzio and F. Rapuano, Physics Letters B 128, 418 (1983).

[12] The ROOT library, http://root.cern.ch/drupal/ . 